Article

\title{
Chaotic Oscillations in a Lipid Doped Membrane
}

\author{
Eduardo J. Delgado * and Cristian Medina \\ Department of Physical-Chemistry. Facultad de Ciencias Químicas, Casilla 160-C, \\ Universidad de Concepción, Concepción, Chile.
}

\begin{abstract}
O potencial elétrico de uma membrana dopada com trioleína e colocada entre duas soluções aquosas de cloretos de sódio e potássio foi analisada para verificar a ocorrência de caos determinístico, usando métodos padrão de teoria de sistemas dinâmicos não lineares. Os resultados fornecem evidências sólidas de que este sistema apresenta dinâmica caótica, e não é apenas um exemplo de ruído experimental.
\end{abstract}

The electric potential of a membrane doped with triolein, placed between aqueous solutions of sodium chloride and potassium chloride, was analyzed for the existence of deterministic chaos using standard methods of nonlinear dynamics system theory. The results provide solid evidence that the system shows a chaotic dynamics and not just an example of experimental noise in the data.

Keywords : chaos, membrane oscillations, excitable lipid membrane, nonlinear dynamics

\section{Introduction}

One of the most interesting phenomena in biological systems is excitability. There is a considerable amount of literature dealing with electrical phenomena associated with excitation in biological membranes, and it has been well established $^{1}$ that differences in the compositions of inorganic cations, especially $\mathrm{Na}^{+}$and $\mathrm{K}^{+}$, inside and outside the membranes are essential for excitability. A number of important biological phenomena are rhytmical in character and appear to result from oscillatory interaction of a membrane with its environment ${ }^{2}$, e.g., oscillations in neurones and secretory glands. The desire to understand the basic physicochemical phenomena that occur during biological membrane oscillations has motivated the interest to study artificial membranes ${ }^{3}$. Although the scale and even the mechanisms of the processes studied may not approximate closely to those in any biological system, all membrane systems have sufficient common structural features to permit knowledge gained with one to be used in considering the behavior of another.

There has been increasing attention paid to aperiodic oscillations, chaos, observed in biological systems. Investigation of chaos in an individual system is, of course, important because of its relationship to other properties of the system concerned. It is probable that characteristics of the system are clarified by the investigation of chaos; for

\footnotetext{
* e-mail: edelgado@udec.cl
}

example, original characteristics of the system may appear in the structure of a chaotic attractor.

Aperiodic oscillations are found at various levels of biological complexity, namely, in a single cell unit such as the giant axon of squids ${ }^{4-9}$; in a more complex element such as in an aggregate of chicken embryo heart cells ${ }^{10}$; and in highly complex systems such as human brain ${ }^{11}$. In almost all cases the oscillations are related to the membrane potential.

In this research, an artificial lipid membrane which is excitable was utilized as a typical model of a self-oscillatory system. The lipid membrane, which is a nitrocellulose filter whose micropores are filled with trioleine (glycerol trioleate), exhibits self-sustained aperiodic oscillations of the membrane potential in the presence of salt concentration difference across the membrane. Previous studies of similar doped-membrane systems have led to inconclusive evidence that the aperiodic behavior observed in this system is an example of chaos. In this study the experimental time series of the membrane potential are analyzed using the methods of Dynamical System Theory, namely, Lyapunov exponent, power spectrum, capacity and correlation dimension. With the present results, we can now state definitively the existence of chaos in this system, and not just an example of experimental noise in the data.

\section{Experimental}

The apparatus used in this study is shown schematically 
in Figure 1. The nitrocellulose filter paper of $0.45 \mu \mathrm{m}$ nominal pore size was obtained commercially from Millipore Corp.(Bedford, MA). The filter paper was immersed in triolein (Sigma Grade approx. 99\%). The membrane was then air-dried and weighed. The adsorbed amount of triolein was determined by measuring the weight change after soaking and drying. Triolein penetrated the filter easily and the quantity absorbed did not depend on the length of immersion time. The quantity of triolein adsorbed in the filter was about $11 \mathrm{mg} \mathrm{cm}{ }^{-2}$. The dried membrane was placed between the circular window of two glass cells. One cell was filled with $0.5 \mathrm{~mol} \mathrm{~L}^{-1} \mathrm{KCl}$ aqueous solution and the other with $0.5 \mathrm{~mol} \mathrm{~L}^{-1} \mathrm{NaCl}$ aqueous solution. The volume of each cell was about $100 \mathrm{~mL}$. The electric potential between the two solutions (membrane potential) was measured with $\mathrm{Ag} / \mathrm{AgCl}$ electrodes inserted in each half-cell. The analog signal from the electrodes was converted to a digital signal through an A/D converter to be collected by the computer via Testpoint ${ }^{\odot}$ (software from Capital Equipment Corp., Millerica MA). The membrane potential readings were done at frequency of 1 $\mathrm{Hz}$ and saved directly to computer disk to be analyzed later by Chaos Data Analyzer ${ }^{\circledR}$ Pro. Version (Physics Academic Software, Raleigh NC). All experiments were conducted at room temperature, $20^{\circ} \mathrm{C}$.

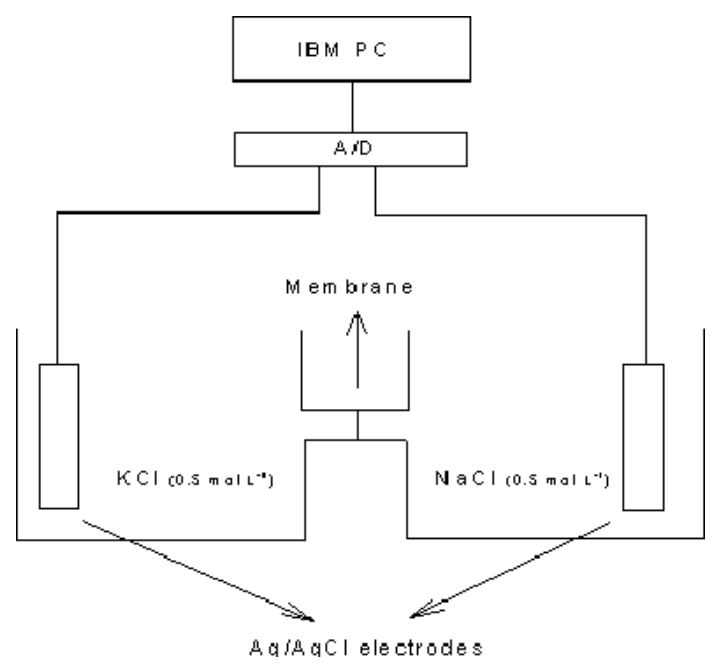

Figure 1. Schematic diagram of the experimental setup.

\section{Results and Discussion}

The experimental data, 682 data points, was filtered, in order to remove the noise that could mask the actual dynamics of the system, using digital filtering functions included in Testpoint ${ }^{\odot}$. The filtering algorithm takes the median of $n$ data points cenrered on the current point. This is a non-linear filter, which eliminates spikes in the data taking the median value of each group of points. The procedure skips the first $(n-1)$ points, until there are enough (n) points available for averaging. In the present study, the value of $n$ was set to 5. A typical time serie of the membrane potential, $\mathbf{X}(\mathrm{t})$, is shown in Figure 2.

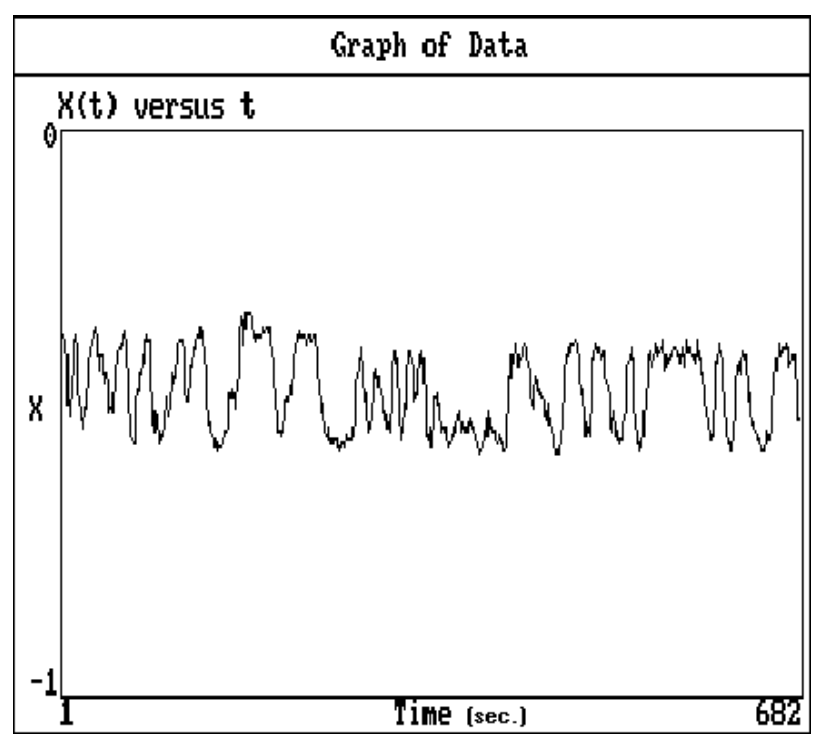

Figure 2. Time series of the membrane potential, $\mathbf{X}(\mathrm{t})$

Any dynamical system at an instant of time can be described by a single point in an appropiate multidimensional phase space. The temporal evolution of the system is then given by the trajectory of that point in phase space. Periodic behavior is given by a closed curve called a limit cycle. A chaotic state is described by an irregular trajectory, called a strange attractor. The phase-space plot constructed from the above time serie is shown in Figure 3. In this two dimensional plot, the time derivative of the membrane potential, $\mathbf{X}^{\prime}(t)$, is plotted versus its value at time $t, \mathbf{X}(\mathrm{t})$. The phase portrait does not show a simple structure suggesting the possible presence of a strange attractor, i.e. a chaotic dynamics. However, this conclusion must wait for more detailed analysis, namely, Lyapunov exponent, correlation and capacity dimension, in order to ascertain with certainty whether the state is truly chaotic.

In order to analyze the experimentally found time series it is sometimes desirable to transform the data from the time into the frequency domain. This particular transformation is called Fourier transformation. The power spectrum which does not carry any phase information is usually used for visualization. Periodic time series give rise to power spectra consisting of discrete lines. There is a main frequency together with its overtones. Quasiperiodic oscillations, instead, show a power spectrum consisting of two non-commensurate frequencies along with their 
overtones. Chaotic dynamics leads to broad banded power spectra made up by a multitude of frequencies. In our case, the power spectrum shows a broad spectrum as expected for chaos, Figure 4.

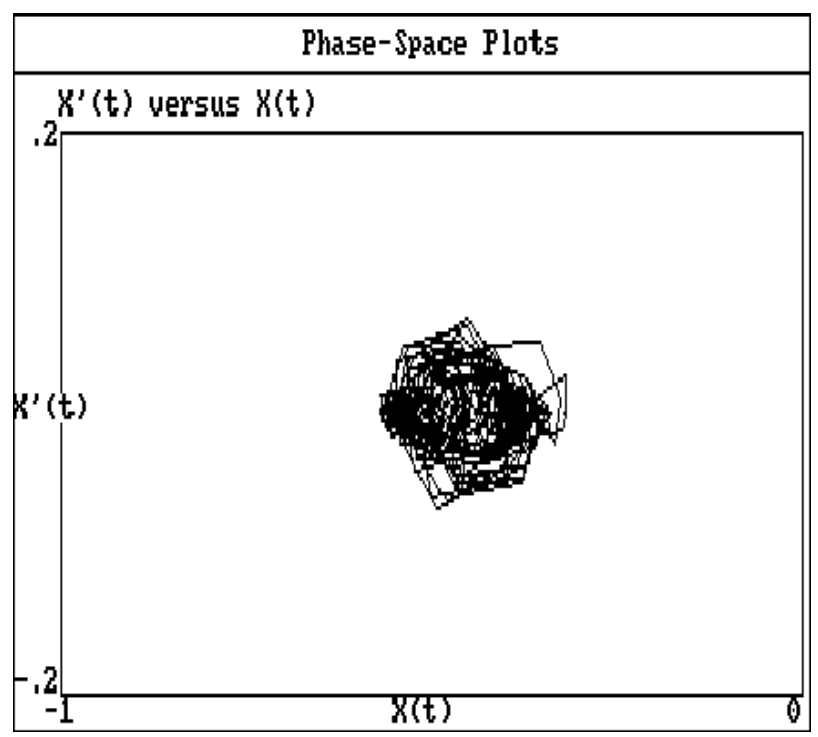

Figure 3. Phase-space plot corresponding to the time series of Figure 2.

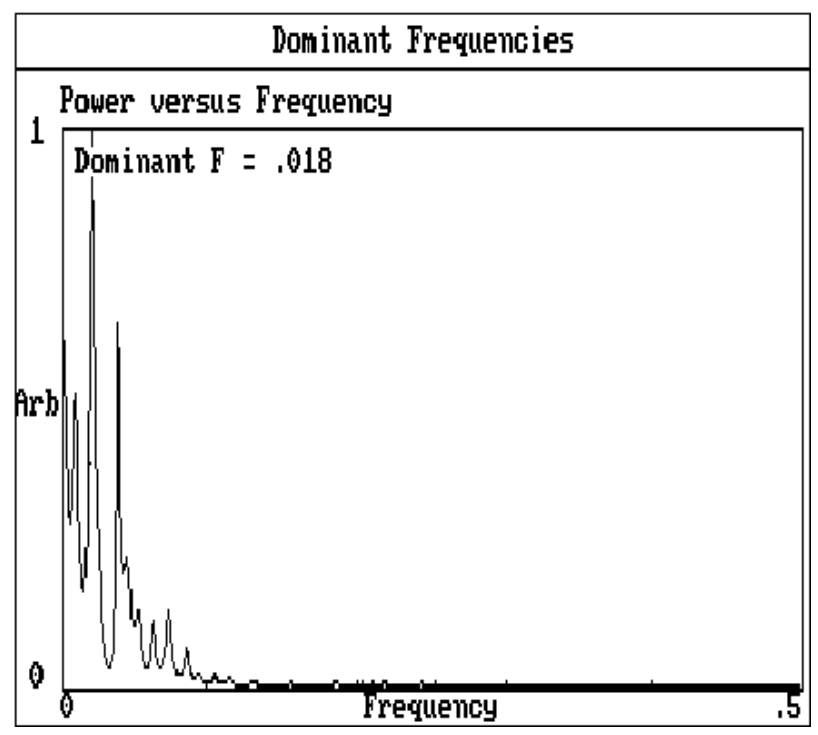

Figure 4. Power spectrum corresponding to the time series of Figure 2.

One of the quantitative measures for chaos is provided by the largest Lyapunov exponent, $\lambda_{\max }$. A positive value of $\lambda_{\max }$ indicates that the trajectories comprising an attractor diverge over a long time average which is characteristic for chaotic motion. This divergence describes the rapid loss of the system's memory of its previous history as time evolves. For periodic motion $\lambda_{\max }$ is equal to zero, indicating that the limit cycle conserves its information in time. The algorithm used in the present study to calculate the largest Lyapunov exponent is due to Wolf et $a l^{12}$. This algorithm involves embedding the time series in an m-dimensional space and monitoring the divergence of trajectories initially close to each other in that phase space. The largest Lyapunov exponent can then estimated from: $\lambda_{\max }=\frac{1}{\mathrm{M} \Delta \mathrm{t}} \sum_{\mathrm{k}=1}^{\mathrm{M}} \log _{2} \frac{\mathrm{d}\left(\mathrm{t}_{\mathrm{k}}\right)}{\mathrm{d}_{\mathrm{o}}\left(\mathrm{t}_{\mathrm{k}-1}\right)}$, where $\mathrm{M}$ is the number of replacement steps and $d_{0}\left(t_{k-1}\right)$ is the separation distance of two initially close points in the trajectory. After a fixed evolution time $\Delta t$ the final distance between the two points evolves to $d\left(t_{k}\right)$. In the present study this figure is +0.572 \pm 0.073 (bits/s), supporting the existence of deterministic chaos.

A powerful tool to characterize an attractor is its dimensionality. Periodic oscillations correspond to a limit cycle in phase space. Since limit cycles represent closed curves their geometric dimension is one. Quasiperiodic oscillations correspond to trajectories covering the surface of a torus; quasiperiodic attractors therefore are characterized by a dimension of two. Strange attractors are self-similar and they do not fill one dimension in embedding space completely. Strange attractors are made up by sets of self-similar trajectories. Therefore, their dimension must be a fractal, noninteger value. As a consequence of the Kaplan-Yorke conjecture ${ }^{13}$ this value must be greater than 2.0. There are two different approaches for the determination of the attractor dimension, one based on the geometric properties, the capacity dimension, of the attractor and the other resulting from a probabilistic point of view, the correlation dimension..

The capacity dimension is calculated using the so-called "box counting" algorithm. In this procedure the phase space with embedding dimension D is successively divided into equal hypercubes and the logarithm of the fraction of hypercubes that are occupied with data points is plotted versus the logarithm of the normalized linear dimension of the hypercubes.

On the other hand, the correlation dimension is computed by the sphere-counting algorithm by Grassberger-Procaccia ${ }^{14}$. Briefly, it is obtained by counting the data points inside hyperspheres of various radii centered on each data point in a reconstructed phase space with some embedding dimension. Its value is close to the capacity dimension, but is generally a more accurate measure.

In this study, the values of the capacity and correlation dimensions are $2.205 \pm 0.591$ and $2.826 \pm 0.156$, respectively (Figures 5 and 6).

The value of the embedding dimension, used for the computation of the largest Lyapunov exponent as well as the dimensions, was set to 3 after the spectrum of eigenvalues of the correlation matrix.

Finally, all the above qualitative and quantitative tests 


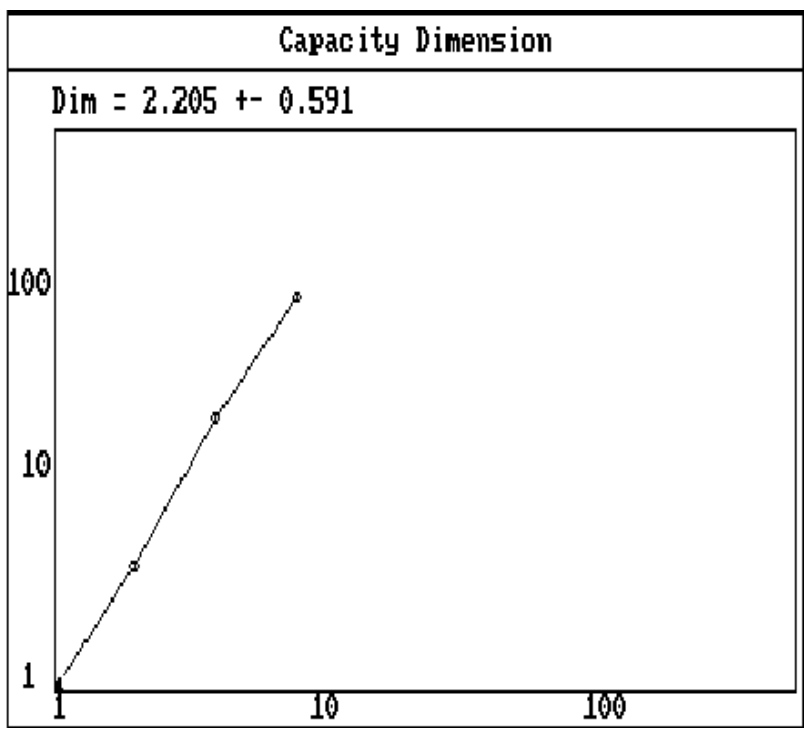

Figure 5. Capacity dimension of the attractor.

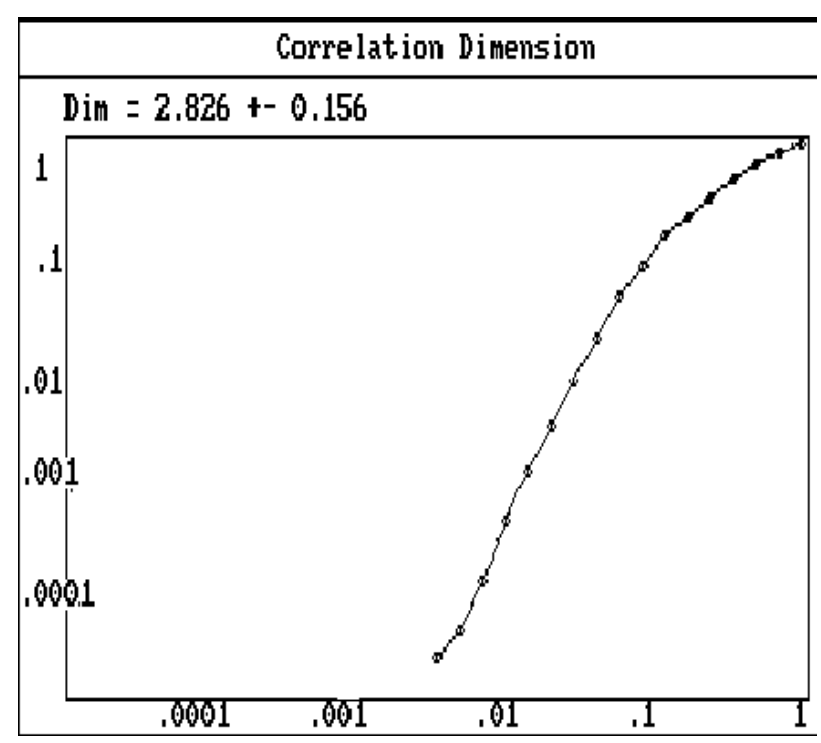

Figure 6. Correlation dimension of the attractor.

provide solid evidence that the aperiodic oscillatory behavior of the membrane potential in the current system, is deterministic chaos, and not just an example of experimental noise in the data. However, it is not yet clear what the decisive factor in determining whether the system becomes chaotic is. The amount of triolein impregnated in the filter seems to play a key role in determining the system dynamics, since the oscillations in the membrane potential are accounted for periodic changes of conductance of the membrane. More experiments under varying conditions will be necessary to answer this question, in particular, temperatures and concentration of solutions will be among the most important parameters to be considered in future studies.

\section{Acknowledgement}

Financial support from Dirección de Investigación, Universidad de Concepción, under grant 99.022.011-1.0 is gratefully acknowledged.

\section{References}

1. Hodgkin, A. L.; Huxley, A. F. J. Physiol. 1952, 117, 500.

2. Chance, B.; Pye, E. K.; Ghosh, A. K.; Hess, B. Biological and Biochemical Oscillators; Academic Press; New York, 1973.

3. Delgado, E. J.; Münster, A. F. J. Braz. Chem. Soc. 1999, 10, 492.

4. Matsumoto, G.; Ahira, K.; Ichikawa, M.; Tasaki, A. J. Theor. Neurobiol. 1984, 3, 1.

5. Aihara, K.; Matsumoto, G.; Ichikawa, M. Phys. Lett. A. 1985, 111, 251.

6. Aihara, K.; Matsumoto, G.; Ikegaya, Y. J. Theor. Biol. 1984, 109, 249.

7. Aihara, K.; Numajiri, T.; Matsumoto, G.; Kotani, M. Phys. Lett. A, 1986, 116, 313.

8. Aihara, K; Matsumoto, G. Chaos.; Holden, A.V. Ed.; Manchester Univ. Press; Manchester, 1986, p. 257.

9. Aihara, K.; Kotani, M.; Matsumoto, G. Structure, Coherence and Chaos in Dynamical Systems; Christiansen, P.L. and Parmentier, R.D. Ed.; Manchester Univ. Press; Manchester, 1989, p. 613.

10. Guevara, M. R.; Glass, L; Shrier, A. Science 1981, 214, 1350.

11. Babloyants, A.; Salazar, J. M.; Nicolis, C. Phys. Lett. A. 1985, 111, 152.

12. Wolf, A.; Swift, J. B.; Swinney, H. L.; Vastano, J. A. Physica 1985, 16 D, 285.

13. Kaplan, J.; Yorke, J. A. Lecture Notes in Mathematics; Peitgen, H. O.; Walter, H. O. Eds.; Springer; Berlin 1978; Vol. 730, p. 228.

14. Grassberger, P.; Procaccia, I. Physica 1983, 9 D, 189.

Received: June 12, 2000

Published on the web: April 2, 2001 